\title{
USO DE ROTEIROS DIDÁTICOS E MODELOS ANATÔMICOS, ALTERNATIVOS, NO ENSINO-APRENDIZAGEM NAS AULAS PRÁTICAS DE ANATOMIA HUMANA
}

\author{
Gleidially Nayara Bezerra MORAES ${ }^{1}$ \\ Paulo Adriano SCHWINGEL ${ }^{2}$ \\ Edivaldo Xavier SILVA JÚNIOR ${ }^{3}$
}

RESUMO:O processo ensino- aprendizagem apresenta- se complexo e difícil no que diz respeito à Anatomia Humana. Com isso, o aprimoramento de recursos didáticos aplicado ao ensino desta disciplina, mostra- se como uma tendência satisfatória e estimula a participação do aluno como sujeito ativo na busca por novas informações, dando suporte indispensável ao processo ensino- aprendizagem. $\mathrm{O}$ objetivo do estudo foi de verificar a existência e utilização de roteiros didáticos e modelos anatômicos nas aulas práticas de Anatomia Humana. O estudo constituiu- se de uma revisão sistemática descritiva, desenvolvida com produção científica indexadas nas bases eletrônicas de dados do LILACS, MEDLINE, GOOGLE ACADÊMICO e SCIELO; assim como em anais de congressos brasileiros. Dentre os 17 artigos encontrados, 9 abordavam o uso de modelos anatômicos, 7 mostravam outros métodos utilizados e apenas 1 sobre a utilização de manual didático em aula prática desta disciplina. A partir do estudo, podese observar que o uso de roteiros didáticos para o ensino em aulas práticas de Anatomia Humana é um método inovador e que a utilização de modelos anatômicos, alternativos, vem mostrando resultados positivos no processo ensino- aprendizagem. Porém, estes métodos, jamais, poderão substituir o uso do cadáver no ensino da referida disciplina.

PALAVRAS-CHAVE: Anatomia humana. Aprendizagem. Ensino.

\section{INTRODUÇÃO}

A Anatomia Humana (AH) é uma disciplina básica para todos os estudantes ingressantes na área de saúde das universidades (KRUSE, 2004). O objetivo desta disciplina é o de compreender a nomenclatura e localização das estruturas específicas do corpo humano, correlacionando-as com as suas funções (BRAZ, 2009).

Atualmente, o ensino tradicional desta disciplina para os cursos de saúde, ocorre através de aulas teóricas predominantemente expositivas, seguidas de aulas práticas em laboratório apropriado. Em contrapartida, as aulas práticas favorecem a visualização, e identificação, das estruturas. Para tanto, o ensino desta disciplina vem sendo visto como um desafio, devido à complexidade das nomenclaturas e a dificuldade de visualização

\footnotetext{
${ }^{1}$ Graduação em Fisioterapia. UPE - Universidade de Pernambuco. Petrolina - PE - Brasil. 56328-903 gleidiallymoraes@ hotmail.com

${ }^{2}$ UPE - Universidade de Pernambuco. Petrolina - PE - Brasil. 56328-903 - paschwingel@ gmail.com

${ }^{3}$ UPE - Universidade de Pernambuco. Petrolina - PE - Brasil. 56328-903 - edivaldo.junior@upe.br
} 
das estruturas, principalmente em aulas práticas, o que dificulta o entendimento para a maioria dos discentes (ARAUJO JUNIOR et al., 2014).

Diante da literatura, é reconhecida a importância de se criar métodos didáticos para o ensino prático da $\mathrm{AH}$, para que haja um maior envolvimento dos alunos e uma maior assimilação e entendimento do conteúdo ministrado (LIMA E SILVA; MACHADO; BIAZUSSI, 2012). Essas novas ferramentas conduzem à melhoria de desempenho do aluno, já que facilitam o aprendizado de conteúdos considerados difíceis de compreender por meio de aulas expositivas, permitindo a construção efetiva do conhecimento (MOTA; MATA; AVERSI-FERREIRA, 2010).

Algumas alternativas vêm apresentando resultados e mostrando- se eficazes no que diz respeito a um maior envolvimento dos discentes nesta disciplina e, consequentemente, um maior aprendizado (LIMA E SILVA; MACHADO; BIAZUSSI, 2012). Dentre elas tem- se o uso de cadáveres humanos dissecados, uma das mais utilizadas para o ensino da AH, assim como o uso de peças sintéticas (COSTA; FEIJÓS, 2009). Porém, faz- se necessário a criação e utilização de novas técnicas e materiais alternativos para o ensino- aprendizagem da AH (MELO; PINHEIRO, 2010).

Nesse contexto, o uso de roteiro didático, assim como de modelos anatômicos para aulas práticas desta disciplina, são métodos que podem ser utilizados para facilitar o entendimento teórico-prático dos discentes, buscando melhores resultados no que diz respeito ao aproveitamento do conteúdo. Sendo assim, o estudo teve como objetivo realizar uma revisão de literatura a fim de verificar a existência e a utilização de roteiros didáticos e modelos anatômicos nas aulas práticas de $\mathrm{AH}$.

\section{METODOLOGIA}

O estudo constituiu- se de uma revisão sistemática descritiva, desenvolvida com produção científica indexadas nas bases eletrônicas de dados do LILACS, MEDLINE, GOOGLE ACADÊMICO e SCIELO; assim como em anais de congressos brasileiros. A pesquisa dos artigos foi realizada entre fevereiro e julho de 2014. A busca nos bancos de dados foi realizada utilizando as terminologias cadastradas nos Descritores em Ciências da Saúde (DECS), como: manual, anatomia, ensino e aprendizagem; associados com outros termos como "métodos didáticos", "modelos anatômicos", "aula prática". A seleção dos artigos foi feita a partir da análise dos títulos e resumos, sendo incluídos os que continham os descritores anatomia e manual, e também os que abordavam a utilização de algum método didático para o ensino da AH. Logo em seguida, foi 
realizada a leitura na íntegra dos artigos selecionados, buscando-se estudar e compreender os métodos didáticos utilizados para o ensino da $\mathrm{AH}$ e sua eficácia no aprendizado dos discentes, e dentre eles, a utilização de roteiros didáticos e modelos anatômicos em aulas práticas.

\section{RESULTADOS E DISCUSSÃO}

Foram encontrados 17 artigos nas bases de dados consultadas que abordavam sobre os metódos didáticos para ensino-aprendizagem da disciplina de $\mathrm{AH}$ e, dentre estes 9 abordavam o uso de modelos anatômicos, 7 mostravam outros métodos utilizados e, apenas 1 sobre a utilização de manual didático em aula prática desta disciplina.

Fornaziero et al. (2010) afirmaram em seu estudo a necessidade de suscitar discussões e reflexões sobre a prática pedagógica para que haja uma melhoria na qualidade do processo de aprendizagem dos futuros profissionais de saúde a partir das reflexões sobre o ensino da AH no contexto atual. Abordando também a importância de incintar avaliações de práticas docentes quanto a utilização de recursos tecnológicos pelo profissional da educação no ensino superior; assim como a inserção de metodologias inovadoras no processo de ensino- aprendizagem, propiciando alternativas para integrar e dinamizar as aulas da referida disciplina.

Adicionalmente, em decorrência das dificuldades em obter materiais de estudo para a disciplina, Montes e Souza (2010) em seu estudo, avaliaram a eficácia da implantação da disciplina optativa "Lições de Anatomia" para os alunos de graduação matriculados no curso de Medicina da Universidade Severino Sombra, no Rio de Janeiro. As aulas ministradas na disciplina envolviam exposição teórica seguida por abordagem prática, enfoncando a necessidade de integrar os conhecimentos anatômicos a situações clínicas. A partir do estudo puderam concluir o quanto é importante a interação docente-discente num processo contínuo de adaptação e de evolução para motivar os alunos a aprenderem de forma significativa, sendo eles os proprios atores do seu aprendizado.

Ademais, com a influência da tecnologia e, como resultado da internet, o uso de métodos modernos de ensino representa um desafio que envolve não só a introdução de tecnologias de informação e comunicação, mas também a interação docente- discente (LEDO et al., 2004). Sendo assim, o desenvolvimento dos recursos multimídia como vídeo aulas, software e verificação dos conteúdos online tornram- se complementos 
úteis aos métodos tradicionais de ensino da AH (GUIRALDES et al., 2001; SAXENA et al., 2008).

Um dos métodos utilizados para o ensino da $\mathrm{AH}$ é o uso de peças cadavéricas, o qual de acordo com Costa e Lins (2012), mesmo que apenas para demonstração de estruturas anatômicas, é considerado indispensável ao processo de ensino-aprendizagem no estudo da $\mathrm{AH}$; e seu manuseio é tido como uma forma de fortalecer a humanização dos futuros profissionais de saúde. Ademais, Lima e Silva, Machado e Biazussi (2012) desenvolveram um projeto com o objetivo de produzir peças anatômicas a partir de órgãos suínos, estimulando a prática de dissecação para os discentes e puderam concluir que esta prática fundamenta o discente de forma mais profunda e duradoura.

Em contrapartida, Carvalho et al. (2010) e Araujo Junior et al. (2014) mostraram que quando não se tem a disponibilidade de cadáveres para o ensino da $\mathrm{AH}$, a confecção e utilização de modelos didáticos sintéticos produzidos com material de baixo de custo pelos próprios discentes é positivo no processo de construção do conhecimento ocasionando uma melhor fixação das estruturas e do conteúdo. Modelos anatômicos são peças tridimensionais similares as peças cadavéricas que podem ser confeccionados pelos próprios alunos, permitindo assim uma melhor visualização e manipulação (LÓPEZ et al., 2011; MAUX et al., 2005; PORTUGAL et al., 2011; SALGADO et al., 2005); além de estimular a criatividade dos discentes e ocasionar uma melhor compreensão, assim como melhoria no processo de ensino- aprendizagem (NAYAK; RODENBAUGH, 2008; NAYAK; SOUMYA, 2009).

Ademais, Inzunza e Salgado (2011) em seu estudo compararam o valor prático do uso de modelos anatômicos com a utilização de peças cadavéricas, e observaram que o desempenho do aluno, com base em avaliação do conteúdo, foi maior quando utilizouse os modelos anatômicos.

Porém, o uso de roteiros didáticos para ensino em aulas práticas de $\mathrm{AH}$ são escassos na literatura, sendo encontrado apenas no estudo desenvolvido por Silva e Brito (2013) que teve por objetivo confeccionar manual de aula prática para o ensino da AH a ser utilizado nas aulas práticas no Departamento de Anatomia Humana da Universidade Federal Rural de Pernambuco. Com a confeccção deste material didático, os referidos pesquisadores alcançaram o objetivo de facilitar e inovar o processo de ensino da $\mathrm{AH}$, efetivando e facilitando o processo de ensino- aprendizagem.

\section{CONCLUSÃO}


$\mathrm{O}$ ensino da $\mathrm{AH}$ tem se tornado um desafio para os docentes, e apesar da existência de alguns métodos alternativos, ainda são escassos os recursos didáticos que venham a estimular e facilitar a aprendizagem dos discentes. A partir do estudo, podese observar que o uso de roteiros didáticos para o ensino em aulas práticas de $\mathrm{AH}$ é um método inovador, visto que é escasso a sua utilização, sua confecção é de baixo custo e sua aplicabilidade facilita o processo ensino- aprendizagem, baseando- se em publicações científicas. Em contrapartida, apesar dos modelos anatômicos serem mais utilizados, sua confeccção e aplicabilidade devem abranger mais universidades, visto que este tipo de material vem mostrando resultados positivos na literatura relacionado a assimilação do conteúdo. Portanto, a utilização de recursos didáticos, alternativos nas aulas de $\mathrm{AH}$, são meios que estimulam o aluno a se interessar pela disciplina. Desta forma, facilita o ensino por parte dos professores, promovendo uma melhoria na qualidade do processo de ensino-aprendizagem nas instituições de ensino superior. Porém, estes métodos, jamais, poderão substituir o uso do cadáver no ensino da referida disciplina.

\section{AGRADECIMENTOS}

A toda a equipe do LABEPAH (Laboratório de Estudo e Pesquisa em Anatomia Humana) da Universidade de Pernambuco, Campus Petrolina.

\section{ALTERNATIVE USES OF DIDACTICSCRIPTS AND ANATOMYMODELSINTHETEACHING- LEARNINGINPRACTICALHUMANANATOMY}


ABSTRACT: The teaching and learning process is complex and difficult presented with respect to the human anatomy. Thus, the improvement of teaching resources applied to the teaching of this discipline, shows up as a satisfactory trend and encourages student participation as an active subject in the search for new informations, giving essential support teaching-learning process. The aim of the study was to verify the existence and utilization of teaching scripts and anatomical models in practicals classes of Human Anatomy. The study was a descriptive systematic review, developed with scientific production indexed in electronic databases LILACS, MEDLINE, GOOGLE ACADEMICO and SciELO; as well as Brazilian proceedings. Among the 17 articles found, 9 showed the use of anatomical models, 7 showed other methods used, and only 1 on the use of didactic manual on classroom practice of this discipline. From the study, it can be observed that the use of teaching scripts for teaching in practical classes of Human Anatomy is an innovative method and the use of anatomical models alternative has shown positive results in the teaching-learning process. However, these methods, ever can replace the use of the corpse in the teaching of this discipline.

KEYWORDS: Human anatomy. Learning.Teaching.

\section{REFERÊNCIAS}

ARAUJO JUNIOR, J. P.et al.Desafio anatômico: uma metodologia capaz de auxiliar no aprendizado de anatomia humana. Medicina, Ribeirão Preto, v.47, n.1, p.62- 68, 2014.

BRAZ, P. R. P. Método didático aplicado ao ensino da anatomia humana. Anuário da produção acadêmica docente, Valinhos, v.3, n.4, p.303-310, 2009.

CARVALHO, D. de O. R. et al. Métodos de ensino aprendizagem em anatomia humana. In: CONGRESSO DE PESQUISA E INOVAÇÃO DA REDE NORTE E NORDESTE DE EDUCAÇÃO TECNOLÓGICA, 5., 2010, Maceió. Anais... Maceió: IFAL, 2010.Disponível em:

<http://connepi.ifal.edu.br/ocs/index.php/connepi/CONNEPI2010/paper/viewFile/453/2 91>. Acesso em: 23 mar. 2016.

COSTA, G. B. F.; LINS, C. C. S. A. O cadáver no ensino da anatomia humana:uma visão metodológica e bioética. Revista Brasileira de Educação Médica, Rio de Janeiro, v.36, n.3, p.369- 373, 2012.

COSTA, L. F.; FEIJÓS, A. G. S. Doação de corpos: estudo comparativo luso-brasileiro sobre a utilização do corpo humano para ensino e pesquisa. In: SALÃO DE INICIAÇÃO CIENTÍFICA, 10., 2009, Porto Alegre. Anais... Porto Alegre: EDIPUCRS, 2009. p.40-42. Disponível em:

<http://www.pucrs.br/edipucrs/XSalaoIC/Ciencias_Biologicas/Bioetica/70839LUCIANA_FRAGA_DA_COSTA.pdf>. Acesso em: 24 mar. 2016.

FORNAZIERO, C. C. et al.O ensino da anatomia: integração do corpo humano e do meio ambiente. Revista Brasileira de Educação Médica, Rio de Janeiro, v.34, n.2, p.290- 297, 2010. 
GUIRALDES, H. et al.Enseñanza de la anatomía humana: experiencias y desafíos en una escuela de medicina. Revista Chilena de Anatomía, Chile, v.19, p.205-212, 2001.

INZUNZA, H. O.; SALGADO, A. G. Evaluaciones prácticas objetivadas en anatomía: diferencias de rendimiento en preguntas realizadas en modelos, preparaciones anatómicas y cadáveres. International Journal of Morphology, Chile, v.29, n.2, p.490-495, 2011.

KRUSE, M. H. L. Anatomia: a ordem do corpo. Revista Brasileira de Enfermagem, Brasília, v.57, n.1, p.79-84, 2004.

LEDO, M. V. et al. Las nuevas tecnologías en la enseñanza y el aprendizaje de la anatomía humana. Educación Médica Superior,Cuba, v.18, n.4, 2004. Disponível em: $<$ http://scielo.sld.cu/scielo.php?script=sci_arttext\&pid=S0864-

21412004000400010\&lng=pt\&nrm=iso\&tlng=es>. Acesso em: 23 mar. 2016.

LIMA E SILVA, M. S.; MACHADO, H. A.; BIAZUSSI, H. M. Produção de material didático alternativo para aula prática de anatomia humana. In: CONGRESSO NORTE NORDESTE DE PESQUISA E INOVAÇÃO, 7., 2012, Palmas. Anais... Palmas: IFTO, 2012. p.1-7. Disponível em:

<http://propi.ifto.edu.br/ocs/index.php/connepi/vii/paper/viewFile/4211/1560>. Acesso em: 24 mar. 2016.

LÓPEZ, F. B. et al. Valoración de la actividad de modelos anatómicos en el desarrollo de competencias en alumnos universitarios y su relación con estilos de aprendizaje, carrera y sexo. International Journal of Morphology, Chile, v.29, n.2, p.568-574, 2011.

MAUX, D. A. S. X. et al. Representação da vascularização do membro superior através de modelo artesanal - recurso adicional às aulas práticas de anatomia. In: REUNIÃO ANUAL DA SOCIEDADE BRASILEIRA PARA O PROGRESSO DA CIÊNCIA, 57., 2005, Fortaleza. Anais... Fortaleza: SBPC, 2005. p.1. Disponível em: <http://www.sbpcnet.org.br/livro/57ra/programas/SENIOR/RESUMOS/resumo_554.ht $\mathrm{ml}>$. Acesso em: 24 mar. 2016.

MELO, E. M.; PINHEIRO, J. T. Procedimentos legais e protocolos para utilização de cadáveres no ensino de anatomia em Pernambuco. Revista Brasileira de Educação Médica, Rio de Janeiro, v.34, n.4, p.315- 323, 2010.

MONTES, M. A. A.; SOUZA, C. T. V. Estratégia de ensino- aprendizagem e anatomia humana para acadêmicos de medicina. Ciências \& Cognição, Rio de Janeiro, v.15, n.3, p.002-012, 2010.

MOTA, M. F.; MATA, F. R.; AVERSI-FERREIRA, T. A. Constructivist pedagogic method used in the teaching of human anatomy. International Journal of Morphology, Chile, v.28, n.2, p.369- 374, 2010.

NAYAK, S. B.; RODENBAUGH, D. W. Modeling the anatomy and function of the pelvic diaphragm and perineal body using a "string model". Advances Physiology Education, Bethesda, v.32, p.169-70, 2008. 
NAYAK, S.; SOUMYA, K.V. A simple model to demonstrate the movements and the axes of the eyeball. Advances Physiology Education, Bethesda, v.33, p.3567, 2009.

PORTUGAL, H. S. P. et al. Modelo pélvico sintético como uma ferramenta didática efetiva comparada à pelve cadavérica. Revista Brasileira de Educação Médica, Rio de Janeiro, v.35, n.2, p.502-506, 2011.

SALGADO, R. F. A. et al. Confecção de modelo artesanal para demonstração da inervação do membro superior: uma inovação no ensino da anatomia. In: REUNIÃO ANUAL DA SOCIEDADE BRASILEIRA PARA O PROGRESSO DA CIÊNCIA, 57. 2005, Fortaleza. Anais... Fortaleza: SBPC, 2005. p.1. Disponível em: <http://www.sbpcnet.org.br/livro/57ra/programas/SENIOR/RESUMOS/resumo_502.ht ml>. Acesso em: 24 mar. 2016.

SAXENA, V. et al. Effect of the use of instructional anatomy videos on students performances. Anatomical Sciences Education, Hoboken, v.1, n.1, p.159-165, 2008.

SILVA, K. R. S.; BRITO, V. C. Manual de aula prática para o ensino de anatomia humana. In: JORNADA DE ENSINO, PESQUISA E EXTENSÃO - JEPEX, 13., 2013, Recife. Anais... Recife: UFRPE, 2013, p.1-3. Disponível em:

<http://www.eventosufrpe.com.br/2013/cd/resumos/r0128-1.pdf>. Acesso em: 24 mar. 2016. 